\title{
SYMBOLS AND DESIGNATIONS FOR GEOMETRICAL OPTICS
}

\author{
By Hermann Kellner
}

I

The arbitrary and often inconsistent way of designating different magnitudes which is practiced by different writers on geometrical optics is confusing to the beginner and annoying to the advanced reader. Every author uses his own system of designation and there is hardly a single one who is not inconsistent.

In accepting the editorship of this journal the possibility occurred to the writer of bringing about, at least in the articles published in this journal, a uniform system of designation and, with this in mind, he approached Dr. Southall for his views on the subject. Dr. Southall has given this matter a good deal of thought, and expressed himself to be in perfect accord with the idea, and as a result of several conferences we offer the following suggestions.

It seemed, in the first place, desirable that the type of the letter give some indication of the nature of the magnitude it represents. We therefore have reserved Roman capital letters for points, lower case italics for magnitudes, italic capitals for reciprocals and Greek lower case letters for angles. Two exceptions from these rules could not be avoided, viz. the use of the $\lambda$ for the wave length and of the $\Delta$ for the optical interval, these departures being justified by universal usage.

The same letter or designation is used for conjugate or similar points or magnitudes on both sides of a refracting surface. They are distinguished from each other by a (') which denotes that the magnitude so marked lies on the side of the optical system away from the side from which the light comes. For instance: $f$ and $f^{\prime}, n$ and $n^{\prime}$ are the focal lengths and refractive indices respectively on the left and the right side of the refracting surface, and $a$ and $a^{\prime}$ the slope angles before and after refraction. Elements of compound system are distinguished by subscripts counting from the left to the right through the system, for instance $r_{1}, r_{2}, r_{3}, \ldots r_{\mathrm{k}}$ would be the first, second, third and $\mathrm{k}^{\text {th }}$ radius of the system. The symbols referring to the whole system in contradistinction to its components are used without subscripts. 
The light is always assumed to enter the optical system from the left side, and the direction from left to right is assumed to be positive.

Distances will be counted positive in the direction of the light, negative in the opposite direction.

Radii are measured from the vertex of the surface to the center. This makes radii of surfaces, convex towards the incident light, positive, while the radii of surfaces concave toward the incident light will be negative.

Object and image distances are measured from the vertex of the surface,

Focal lengths from the principal points.

Ordinates in object and image planes are positive above and negative below the axis.

Slope angles above the axis are positive when they converge in the direction of the light, negative when they diverge. They take the opposite sign when below the axis.

Designations of Points (Roman capital letters)

Focal points

$\mathrm{F}$ and $\mathrm{F}^{\prime}$

Principal points

$\mathrm{H}$ and $\mathrm{H}^{\prime}$

Nodal points

$\mathrm{N}$ and $\mathrm{N}^{\prime}$

Conjugate object and image points

$\mathrm{O}$ and $\mathrm{O}^{\prime}$

Centre of spherical refracting surface

C

Vertex of spherical surface

Symbols of Magnitudes (Italic lower case)

Index of refraction

Wavelength

Radius of curvature of refracting system

Focal lengths of optical system

Distance from vertex $\mathrm{V}$ to focal point $\mathrm{F}$

Distance between vertices of two refracting surfaces

Distance between vertex of refracting surface and object or image point

Incidence height

Abscissae measured from principal points

Reduced distance between two principal planes

Abscissae measured from focal points

Ordinates of conjugate points

$n, n^{\prime}$
$\lambda$
$r$
$f$ and $f^{\prime}$
$v$ and $v^{\prime}$
$d$
$u$ and $u^{\prime}$
$h$
$b$ and $b^{\prime}$
$c$
$x$ and $x^{\prime}$
$y$ and $y^{\prime}$


Symbols of Magnitudes (Italic lower case) Continued

In a compound system the distance between the posterior focal point of the first component and the anterior focal point of the second component, the optical interval,

Distance between the focal point of a compound system and the posterior focal point of the second component

Reciprocals of magnitudes

are to be designated in the corresponding capital italics thus:

$$
U=\frac{\mathrm{I}}{u} \quad X^{\prime}=\frac{\mathrm{I}}{x^{\prime}} \quad F=\frac{\mathrm{I}}{f} \quad V=\frac{\mathrm{I}}{v}
$$

or better still, following Gullstrand, let

$$
U=\frac{n}{u} \quad X^{\prime}=\frac{n}{x^{\prime}} \quad F=\frac{n}{f} \quad V=\frac{n}{v}
$$

denoting by capital italic letters the so-called reduced lengths.

$F$ and $V$ represent quantities of great importance in ophthalmic optics, $F$ is the so-called refraction of a lens, $V$ its vertex refraction. Both are expressed in diopters (dptr) when $f$ and $v$ are measured in meters.

Angular magnitudes are denoted by lower case Greek letters.

Slope angle

Angle of incidence and refraction (or reflection)

Central angle

Magnifications

Lateral magnification

Axial or Depth magnification

Angular magnification $a$ and $a^{\prime}$

$\vartheta$ and $\vartheta^{\prime}$

$\varphi$

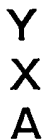

$A$

The following diagrams are given to supplement the foregoing list of designations. 


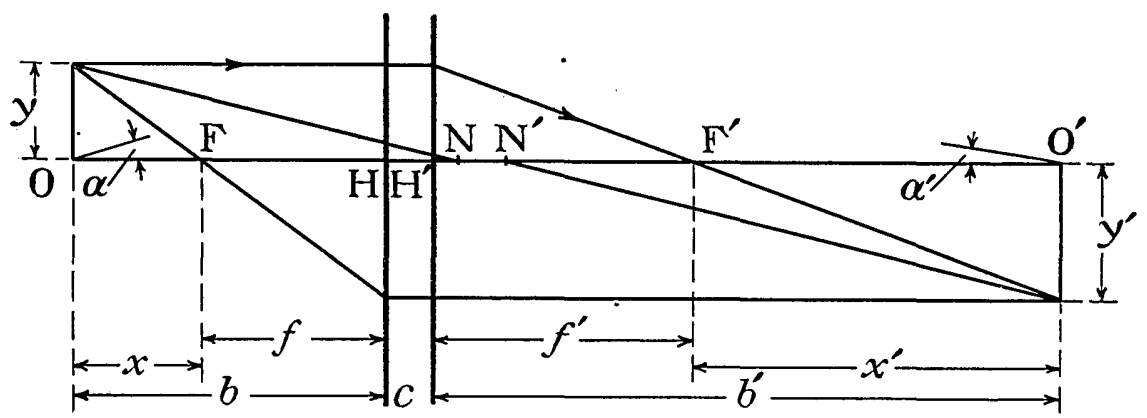

Fig. 1

An object $y$, imaged by an optical system represented by the principal points $H$ and $H^{\prime}$, the nodel points $N$ and $N^{\prime}$, and the focal points $F$ and $F^{\prime}$.

$$
x \cdot x^{\prime}=f \cdot f^{\prime}
$$

$$
\begin{gathered}
\frac{\mathrm{d} x^{\prime}}{\mathrm{d} x}=-\frac{f \cdot f^{\prime}}{x^{2}}=-\frac{x^{\prime}}{x}=\mathrm{X} \\
\frac{y^{\prime}}{y}=\frac{f}{x}=\frac{x^{\prime}}{f^{\prime}}=\mathrm{Y} \\
\frac{\tan a^{\prime}}{\tan a}=-\frac{x}{f^{\prime}}=-\frac{f}{x^{\prime}}=\mathrm{A} \\
y^{\prime} n^{\prime} \tan a^{\prime}=y n \tan a
\end{gathered}
$$$$
Y \mathrm{~A}=\frac{n}{n^{\prime}}=\frac{f}{f^{\prime}}
$$$$
\frac{X A}{Y}=1
$$

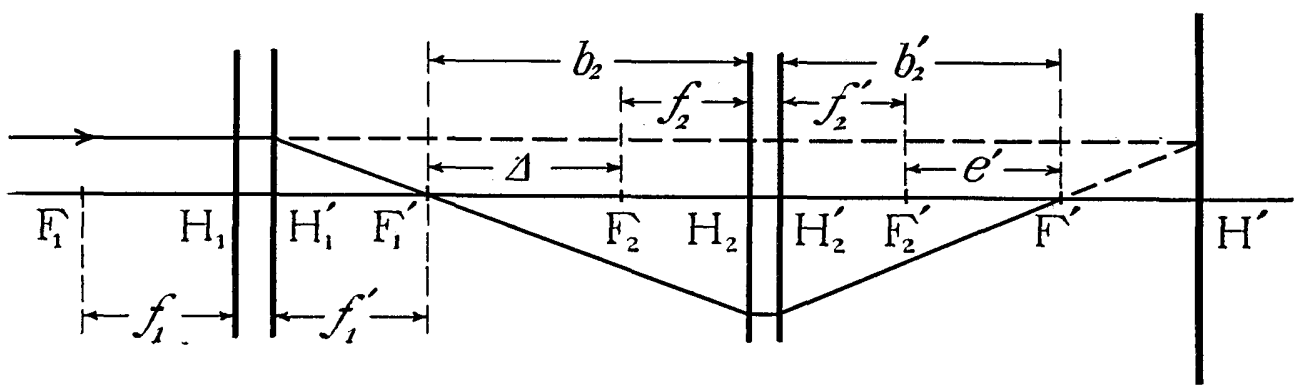

Fig. 2

A compound system composed of two elements represented by the principal points $\mathrm{H}_{1}, \mathrm{H}_{1}{ }^{\prime}$ and $\mathrm{H}_{2}, \mathrm{H}_{2}{ }^{\prime}$, and by the focal points $\mathrm{F}_{1}, \mathrm{~F}_{1}{ }^{\prime}$ and $\mathrm{F}_{2}, \mathrm{~F}_{2}{ }^{\prime}$, the distance $\mathrm{F}_{1}{ }^{\prime}, \mathrm{F}_{2}$ being the optical interval $\Delta$; object point at $-\infty$.

$$
\begin{gathered}
f^{\prime}=\frac{f_{\mathrm{I}}{ }^{\prime} f_{2}}{\Delta} \quad f=-\frac{f_{\mathrm{I}} f_{2}{ }^{\prime}}{\Delta} \\
e^{\prime}=-\frac{f_{2} \cdot f_{2}{ }^{\prime}}{\Delta} \\
f^{\prime}=b^{\prime} \text { because } u=\infty
\end{gathered}
$$




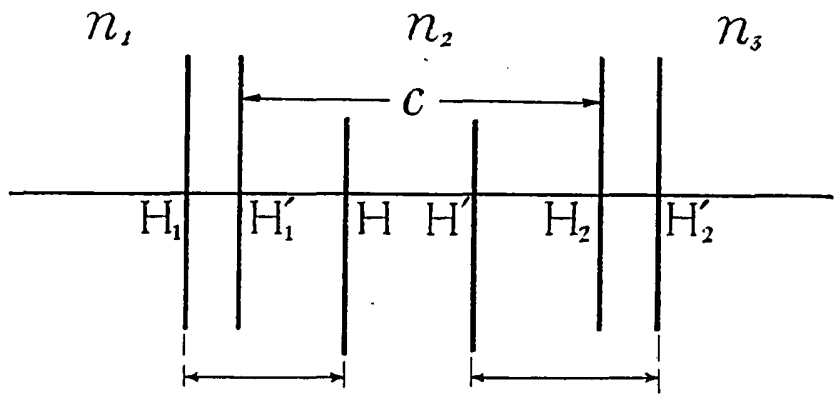

Fig. 3

The principal planes $\mathrm{H}, \mathrm{H}^{\prime}$ of a compound system, and those of its components $\mathrm{H}_{1}, \mathrm{H}_{1}{ }^{\prime}$ and $\mathrm{H}_{2}, \mathrm{H}_{2}{ }^{\prime}$ in their relative locations.

The refracting power of a compound system (Fig. 3) composed of the components having respectively the powers $F_{1}$ and $F_{2}$ and having a separation between their principal points $\mathrm{H}_{\mathrm{r}}{ }^{\prime}$ and $\mathrm{H}_{2}$ equal to $c$, will be

where $c=\frac{\mathrm{H}_{\mathrm{I}} \mathrm{H}_{2}}{n_{2}}$.

$$
F=F_{1} F_{2}-F_{1} F_{2} c
$$

The location of the principal points of the whole system with respect to $\mathrm{H}_{1}$ and $\mathrm{H}_{2}{ }^{\prime}$ follows from

$$
\frac{\mathrm{H}_{\mathrm{I}} \mathrm{H}}{n_{\mathrm{I}}}=c \frac{F_{2}}{F} \quad \frac{\mathrm{H}_{2}{ }^{\prime} \mathrm{H}^{\prime}}{n_{3}}=-c \frac{F_{\mathrm{I}}}{F}
$$

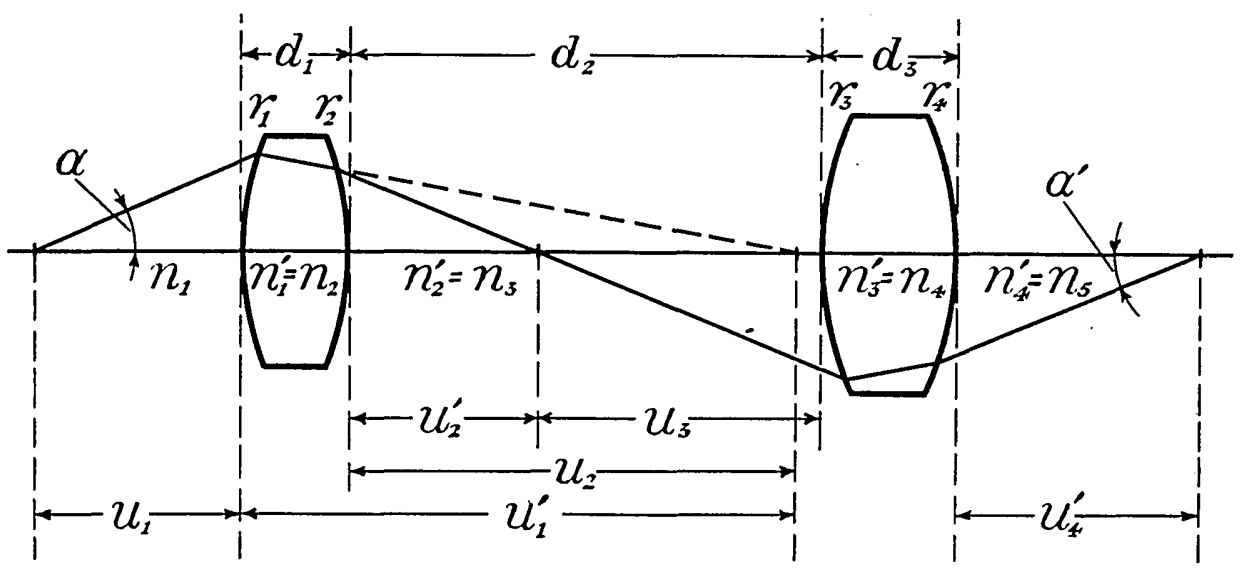

Fig. 4

Optical imagery through a compound system consisting of two positive lenses of thicknesses $\mathrm{d}_{1}$ and $\mathrm{d}_{3}$ respectively, separated by a distance $\mathrm{d}_{2}$.

In Fig. 4 is

$$
\begin{aligned}
& u_{2}=u_{\mathrm{r}}^{\prime}-d_{\mathrm{I}} \\
& u_{3}=u_{2}^{\prime}-d_{2} \text { etc. } \\
& n_{1}^{\prime}=n_{2} \\
& n_{2}^{\prime}=n_{3} \text { etc. } \\
& \text { when } u_{\mathrm{I}}=\infty, \quad u_{4}^{\prime} \text { becomes } v^{\prime} \text {. }
\end{aligned}
$$




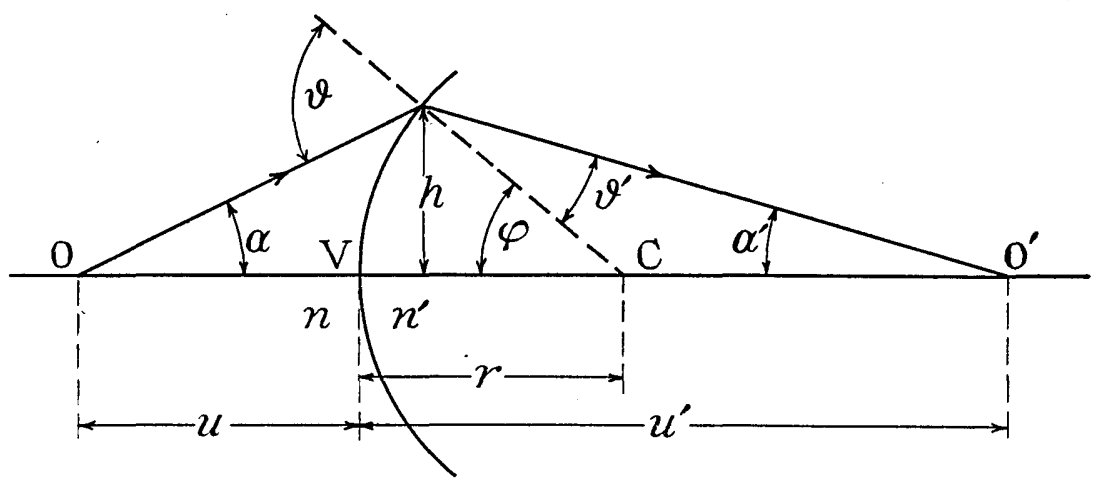

Fig. 5

Designation of the magnitudes essential in trigonometric calculation of the path of a ray refracted by a spherical surface, the ray path lying in the plane of a principal section.

Fig. 5 is self-explanatory and requires no comment.

(To be continued)

\section{Optical Society of America, Annual Meeting, New York City, December 28, 1916}

The first Annual Meeting of the Optical Society was held in New York City, December 28, in Room 30I of the Physics Building, Fayerweather Hall, under the auspices of the A. A. A. S., and the following papers were read:

L. T. Troland, Harvard University.

Notes on the Theory of the Visual Receptor Process.

M. B. Hodgson, Eastman Kodak Co.

Compensating Filters for Use in Spectroscopy.

Edw. P. Hyde and W. E. Forsythe, Nela Research Laboratory.

The Quality of Light Emitted by Various Sources as Indicated by Their Color Temperatures.

F. E. Ross, Eastman Kodak Co.

I) The Photography of Star Images.

*2) The Effects of Exposure, Developer and Development on Photographic Resolving Power.

E. L. Nichols, Cornell University.

Optical Research.

F. E. Wright, Geophysical Laboratory.

I) The Petrographic Microscope in Applied Optics.

2) The Determination of Anisotropism in Opaque Substances.

H. C. Lord, Emerson McMillan Observatory.

On Designing a Symmetrical Photographic Doublet Consisting of Four Separated Lenses.

Herbert E. Ives, United Gas Improvement Co.

The Use of Lippmann Films as Sources of Monochromatic Light in Photometry and Optical Pyrometry.

H. Kellner, Bausch \& Lomb Optical Co.

The Determination of the Chromatic Aberration of the Eye for Different Zones of the Pupil.

L. A. Jones, Eastman Kodak Co.

The Fundamental Hue Scale and the Retinal Sensibility to Hue Differences.

${ }^{*}$ C. W. Frederick, Eastman Kodak Co.

Variants of the Seidel Aberrations in the Four Piece Separated Type of Objective.

Julian Blanchard, Eastman Kodak Co.

The Brightness Sensibility of the Retina.

P. G. Nutting, Westinghouse Research Laboratory.

1) A Photochemical Theory of Vision and Photographic Action.

2) A Proposed New Method of Classification for Optical Glass.

* Read by title. 